\title{
Eficacia de las medidas de bioseguridad en el control de microorganismos asociados a endometritis porcinas. Estudio preliminar
}

\author{
Efficacy of biosecurity measures in the control of microorganisms associated \\ to endometritis in sows. Preliminary study \\ JC Gómez, B Huerta, I Luque, A Maldonado, RJ Astorga, C Tarradas* \\ Departamento de Sanidad Animal, Facultad de Veterinaria, Universidad de Córdoba, Córdoba, España.
}

\begin{abstract}
SUMMARY
Biosecurity can be defined as all the applied measurements that take as a target to minimize the sanitary risks in a stock farm, and include measurements related to the facilities and the management. The efficacy of these measurements must be reflected in a decrease of the microorganism in different productive phases. A study was carried out to evaluate if the set of applied measurements influences the microbial uterine contamination after farrowing of healthy sows. Two swine farms were been completed about biosecurity measurements was completed and a microbiological study of uterine swabs of sows after the farrowing was carried out. A total of 60 animals were studied, and 27 (45\%, 95\% CI [33.3\%, 56.7\%]) resulted positive. Significant differences between production and selection and multiplication farms were detected (OR $=3.44$, IC 95\%, 1.135-11.047). The colonization frequency was $65 \% \mathrm{CI}[51.3 \%, 78.6 \%]$ and $35 \% \mathrm{CI}[21 \%, 49 \%]$ in production and selection farm, respectively $(\mathrm{P}=0.02)$. A total of 66 isolates were obtained, represented mainly by Staphylococcus spp. (33.33\%) and Aerococcus spp. (27.27\%), although other species included in the genus Streptococcus (9.09\%), Enterococcus $(6.06 \%)$ and Pseudomonas (4.55\%), as well as different fungi species were also isolated. The frequency of isolation of different microorganisms was similar in both farms, with the exception of the genus Enterococcus that was not isolated in the production farm $(\mathrm{P}=0.01)$. The questionnaire showed some differences in biosecurity measures in the selection and multiplication farm when it is compared to the production farm, which together with the increased uterine microbial contamination observed in the latter leads us to propose a preliminary hypothesis about the possible risk factors associated with this process, highlighting the absence of measures to avoid the presence of vectors and the establishment of strict protocols for cleaning and disinfection.
\end{abstract}

Palabras clave: bioseguridad, endometritis, cerdas.

Key words: biosecurity, endometritis, sow.

\section{INTRODUCCIÓN}

El concepto de bioseguridad hace referencia al mantenimiento de granjas con una carga mínima de microorganismos que no interfiera en la obtención de una máxima rentabilidad ganadera. Podemos pues definir la bioseguridad en este contexto como el conjunto de medidas encaminadas a reducir la entrada y diseminación de agentes patógenos, y sus vectores, en las granjas (Quiles 2008).

La endometritis se puede definir como la inflamación del endometrio, y en muchas ocasiones está producida por microorganismos saprofíticos, cuya presentación está ligada al manejo, condiciones higiénicas y sanitarias de las explotaciones (Quiles y Hevia 2006). Un cuadro de endometritis se puede desarrollar tras finalizar el parto, cuando los microorganismos penetran en el útero a través del cérvix abierto y no son eliminados por la inmunidad local de la hembra (Rodríguez y col 1989a,b). Los microorganismos asociados a las endometritis porcinas son muy variados, tanto de etiología fúngica como bacteriana, y están representados principalmente por los grupos de enterobacterias,

Aceptado: 20.10.2010.

* Campus Universitario de Rabanales, 14071 Córdoba, España; sa1taigc@uco.es estreptococos, estafilococos y corinebacterias (Rodríguez y col 1989 $9^{\mathrm{a}, \mathrm{b}}$, Bara y col 1993, Falceto y Úbeda 2008).

Las medidas de bioseguridad en las granjas porcinas deben aplicarse de forma preventiva para evitar la entrada de agentes patógenos que puedan afectar a la sanidad, bienestar y producción de los animales (Quiles y Hevia 2006). Por lo tanto, el principal objetivo de este trabajo es determinar de forma preliminar si la implementación de medidas de bioseguridad relacionadas con las instalaciones, control de roedores y otros vectores, programas de limpieza y desinfección, así como la utilización de un código de buenas prácticas en las granjas, pueden influir en el nivel de contaminación del útero tras el parto. Para ello se estudiarán las medidas de bioseguridad en dos granjas porcinas en España, realizando posteriormente un estudio microbiológico sobre hisopos uterinos obtenidos de hembras aparentemente sanas.

\section{MATERIAL Y MÉTODOS}

\section{MATERIAL PATOLÓGICO}

El estudio se realizó en dos granjas porcinas situadas en España, cuyos dueños aceptaron participar en el trabajo previo consentimiento informado. La primera de 
las explotaciones se dedicaba a la producción de cerdas de raza ibérica $(n=35)$, utilizaba reposición externa y realizaba las cubriciones mediante monta natural. Las granjas de producción se definen, según la legislación española, como aquellas explotaciones que en una sola unidad productiva o utilizando el sistema de producción en fases, están dedicadas a la producción de lechones para su engorde y sacrificio, pudiendo generar sus reproductores para la autorreposición (Boletín Oficial del Estado 2000). La segunda explotación se dedica a la selección y multiplicación intensiva de cerdas de razas puras o híbridas $(n=400)$, realizaba reposición propia y aplicaba inseminación artificial. Las granjas de selección y multiplicación se definen en la legislación española como aquellas dedicadas a la multiplicación de animales de razas o estirpes selectas, puras o híbridas, procedentes de las explotaciones de selección, cuya finalidad principal es la obtención de animales destinados a la reproducción, mediante la aplicación de los correspondientes programas zootécnicos y sanitarios, pudiendo generar sus reproductores para la autorreposición (Boletín Oficial del Estado 2000).

Para determinar la frecuencia de aislamiento de microorganismos a partir del útero por microorganismos potencialmente patógenos entre 3-5 días postparto, se calculó el número mínimo de muestras a analizar en base al tamaño de las granjas, una prevalencia esperada del 78,3\% (Bara y col 1993), un error máximo aceptado del $12 \%$ y una seguridad del $95 \%$. Se analizaron un total de 20 muestras en la explotación de producción y 40 en la explotación de multiplicación y selección.

Todas las muestras analizadas fueron obtenidas a partir del útero, entre 3-5 días postparto, mediante hisopos estériles con medio de transporte de Amies (EUROTUBO ${ }^{\circledR}$, Deltalab) y enviadas al Laboratorio de Diagnóstico del Departamento de Sanidad Animal de la Facultad de Veterinaria (Universidad de Córdoba, España) donde fueron procesadas inmediatamente.

\section{ESTUDIO MICROBIOLÓGICO}

Los hisopos se sembraron en medios de cultivo específicos para el aislamiento de los posibles microorganismos presentes en la mucosa uterina de las cerdas: agar Columbia, adicionado de sangre de ovino desfibrinada y estéril, y un suplemento de ácido nalidíxico y colistina, para el aislamiento de estreptococos; agar Xilosa Lactosa Desoxicolato (XLD) y agar MacKonkey, para el aislamiento de bacterias Gram negativas, agar Manitol, para el aislamiento selectivo de estafilococos y micrococos y agar Sabouraud, adicionado de un suplemento antibiótico de cloranfenicol, para el aislamiento de levaduras y hongos filamentosos. Todos los medios fueron suministrados por casas comerciales (Oxoid, Ltd.) y preparados siguiendo las instrucciones del fabricante.
Tras la siembra, las placas se incubaron a una temperatura de $37^{\circ} \mathrm{C}$ durante $18-24 \mathrm{~h}$ en condiciones de aerobiosis; las placas de agar Sabouraud se mantuvieron en la estufa durante un tiempo más prolongado (72 h). Se consideró un resultado positivo cuando crecieron más de diez colonias del mismo tipo en el medio (Fthenakis 1994). Como se ha indicado anteriormente, uno de los objetivos de nuestro trabajo era determinar el nivel de contaminación del útero tras el parto.

La identificación de todos los microorganismos aislados se realizó mediante pruebas bioquímicas convencionales y comerciales (API 20 STREP, API STAPH, API E, API NO E, API CORYNE, API 20AUX, Biomeriux, S.A). La lectura de los resultados se realizó con perfiles numéricos procesados mediante la aplicación informática apiweb ${ }^{\circledR}$ (BioMérieux).

\section{ENCUESTA EPIDEMIOLÓGICA}

En el momento de la toma de muestras los veterinarios completaron un cuestionario donde se chequearon los principales puntos críticos de bioseguridad de las explotaciones porcinas. Esta encuesta recoge las medidas de bioseguridad contempladas en las Guías de Buenas Prácticas emitidas por el Ministerio de Medio Ambiente, Medio Rural y Marino del Gobierno de España (http:// rasve.mapa.es). Este protocolo recoge los principales puntos críticos de bioseguridad relacionados con: (i) instalaciones e infraestructura; (ii) protocolos de limpieza y desinfección; (iii) control de vectores, y otros animales. Este cuestionario ha sido completado por los veterinarios responsables de las granjas.

La comparación de ambos cuestionarios nos permitiría obtener información preliminar sobre las posibles medidas de bioseguridad, presentes en una explotación y ausentes en la otra, que podrían influir en el riesgo de colonización uterina por microorganismos potencialmente patógenos, de cara a la futura realización de un estudio analítico sobre factores de riesgo.

\section{ANÁLISIS ESTADÍSTICO DE LOS DATOS}

En base a los resultados del estudio microbiológico se determinó la frecuencia de aislamiento del útero de las cerdas en ambas granjas y su intervalo de confianza. La significación estadística de las diferencias encontradas se estimó mediante el test de Chi-cuadrado $\left(\chi^{2}\right)$, para un valor de $\mathrm{P}<0,05$. Los cálculos estadísticos se han realizado utilizando el programa SPSS v.15 para Windows.

\section{RESULTADOS Y DISCUSIÓN}

La producción porcina ha experimentado en los últimos años una gran transformación; hoy en día alcanzar una elevada rentabilidad supone conseguir niveles productivos adecuados, garantizando la calidad y salubridad de los 
productos obtenidos. En este sentido, el técnico veterinario debe actuar reduciendo el riesgo de infección en las granjas, aplicando correctas medidas de bioseguridad en todos los eslabones de la cadena productiva y controlando la eficacia de las mismas.

En este trabajo se han analizado las encuestas de bioseguridad completadas por los veterinarios responsables de dos explotaciones, para determinar la posible influencia de la aplicación de estas medidas en el número y diversidad de microorganismos aislados a partir de hisopos uterinos de cerdas tras el parto. Comparando los resultados de las dos encuestas podemos observar que existen un número más elevado de medidas generales de bioseguridad para reducir posibles riesgos sanitarios en la granja de selección y multiplicación frente a la granja de producción.
Según se observa (cuadro 1), se detectan deficiencias en la granja de producción que permiten la posible entrada de agentes patógenos. En primer lugar, en cuanto a las medidas de carácter general relacionadas con el tipo de instalaciones y condiciones externas, podemos comprobar que a pesar de que las naves presentaban un aspecto adecuado de mantenimiento y limpieza, en la granja de producción no se controlaba la presencia de cubierta vegetal, lo que supone un foco potencial de atracción para animales peridomésticos (aves, roedores y carnívoros silvestres). Destacamos asimismo la ausencia de duchas en la cámara de entrada, así como de pediluvios o bandejas de desinfección a la entrada de las naves, e incluso la inexistencia de controles en las visitas a la explotación. Por otro lado, entre las medidas relacionadas

Cuadro 1. Encuesta de bioseguridad completada por los veterinarios en las dos granjas analizadas. Biosafety questionary filled by the veterinarians of both selected farms.

\begin{tabular}{|c|c|c|c|c|}
\hline \multirow[b]{3}{*}{ 1. Medidas generales de bioseguridad } & \multicolumn{4}{|c|}{ Explotaciones } \\
\hline & \multicolumn{2}{|c|}{ Producción } & \multicolumn{2}{|c|}{ Selección } \\
\hline & Sí & No & Sí & No \\
\hline Vallado perimetral & $\mathrm{X}$ & & $\mathrm{X}$ & \\
\hline Puerta entrada visible & $\mathrm{X}$ & & $\mathrm{X}$ & \\
\hline Vado de desinfección / arco de desinfección / otros sistemas & & $\mathrm{X}$ & $\mathrm{X}$ & \\
\hline Distancia $>1 \mathrm{~km}$ a otras granjas & $\mathrm{X}$ & & $\mathrm{X}$ & \\
\hline Distancia $>0,5 \mathrm{~km}$ a vías públicas & $\mathrm{X}$ & & & $\mathrm{X}$ \\
\hline Cubierta vegetal perimetral en entorno de naves & $\mathrm{X}$ & & & $\mathrm{X}$ \\
\hline Limpieza y conservación exterior de la nave & $\mathrm{X}$ & & $\mathrm{X}$ & \\
\hline Vestuarios a la entrada de naves (botas, calzas, overol) & & $\mathrm{X}$ & $\mathrm{X}$ & \\
\hline Duchas cámara de entrada & & $\mathrm{X}$ & $\mathrm{X}$ & \\
\hline Acceso a naves mediante pediluvios/bandejas de desinfección & & $\mathrm{X}$ & $\mathrm{X}$ & \\
\hline Contenedores para material desechable & & $\mathrm{X}$ & $\mathrm{X}$ & \\
\hline Control de acceso de visitas: libro de registro de visitas & & $\mathrm{X}$ & $\mathrm{X}$ & \\
\hline Instalaciones para cuarentena & & $\mathrm{X}$ & $X$ & \\
\hline Lazareto & $\mathrm{X}$ & & $\mathrm{X}$ & \\
\hline Telas mosquiteras & & $\mathrm{X}$ & $\mathrm{X}$ & \\
\hline Mallas antipájaros & $\mathrm{X}$ & & $\mathrm{X}$ & \\
\hline Ventilación natural/forzada & $\mathrm{X}$ & & $\mathrm{X}$ & \\
\hline Agua de red, o clorada o tratamiento equivalente & & $\mathrm{X}$ & $\mathrm{X}$ & \\
\hline Análisis de agua periódicos y documentados & $\mathrm{X}$ & & $\mathrm{X}$ & \\
\hline Silos cerrados & $\mathrm{X}$ & & $X$ & \\
\hline Silos: limpieza y fumigación & & $\mathrm{X}$ & $X$ & \\
\hline Certificados de análisis periódicos de pienso (proveedores) & & $\mathrm{X}$ & $X$ & \\
\hline Se emplean aditivos autorizados (acidificantes) & $\mathrm{X}$ & & $\mathrm{X}$ & \\
\hline Almacén de piensos protegido ( $\mathrm{T}^{\mathrm{a}}$ refrigeración) & & $\mathrm{X}$ & & $\mathrm{X}$ \\
\hline Muelles de embarque de animales & $\mathrm{X}$ & & $\mathrm{X}$ & \\
\hline Existe un sistema adecuado de retirada de estiércol & $\mathrm{X}$ & & $\mathrm{X}$ & \\
\hline Existe un sistema adecuado de retirada de purines & $\mathrm{X}$ & & $\mathrm{X}$ & \\
\hline Fosa de purines & $\mathrm{X}$ & & $\mathrm{X}$ & \\
\hline Existe un sistema de gestión/eliminación de cadáveres & $\mathrm{X}$ & & $X$ & \\
\hline
\end{tabular}


Cuadro 1. (Continuación)

\begin{tabular}{|c|c|c|c|c|}
\hline \multirow[b]{3}{*}{ 2. Protocolo de limpieza, desinfección y desinsectación } & \multicolumn{4}{|c|}{ Explotaciones } \\
\hline & \multicolumn{2}{|c|}{ Producción } & \multicolumn{2}{|c|}{ Selección } \\
\hline & Sí & No & Sí & No \\
\hline Existe registro de programa de limpieza + DD & & $\mathrm{X}$ & $\mathrm{X}$ & \\
\hline Se realizan análisis de eficacia de tales programas & & $\mathrm{X}$ & & $\mathrm{X}$ \\
\hline Los operarios conocen el procedimiento & $\mathrm{X}$ & & $\mathrm{X}$ & \\
\hline Se sigue el sistema todo dentro, todo fuera & $\mathrm{X}$ & & $\mathrm{X}$ & \\
\hline Se respeta el periodo mínimo de vacío sanitario & $\mathrm{X}$ & & $\mathrm{X}$ & \\
\hline Se utilizan productos homologados (MAPA) & $\mathrm{X}$ & & $\mathrm{X}$ & \\
\hline \multicolumn{5}{|l|}{ 3. Protocolo de control de roedores y otros animales } \\
\hline Existe un plan de desratización documentado & & $\mathrm{X}$ & $\mathrm{X}$ & \\
\hline Por medios propios (cebos, trampas) & $X$ & & & $\mathrm{X}$ \\
\hline Por empresas autorizadas (cebos, trampas) & & $\mathrm{X}$ & $X$ & \\
\hline Existen agujeros en instalaciones que permitan el paso & & $\mathrm{X}$ & & $\mathrm{X}$ \\
\hline Los perros/gatos están controlados (acceso a naves) & $\mathrm{X}$ & & $\mathrm{X}$ & \\
\hline Los perros/gatos están desparasitados y vacunados & $\mathrm{X}$ & & $\mathrm{X}$ & \\
\hline \multicolumn{5}{|l|}{ 4. Protocolo de Control Sanitario y Buenas Prácticas } \\
\hline Existe Libro de Registro & $\mathrm{X}$ & & $\mathrm{X}$ & \\
\hline Existe un veterinario que realice visitas y controles & $\mathrm{X}$ & & $\mathrm{X}$ & \\
\hline Existen planes de vacunación y desparasitación & $\mathrm{X}$ & & $\mathrm{X}$ & \\
\hline Existe un Código de Buenas Prácticas de Higiene & & $\mathrm{X}$ & & $\mathrm{X}$ \\
\hline Los operarios están formados, o existe protocolo & & $\mathrm{X}$ & & $\mathrm{X}$ \\
\hline Vestimenta adecuada en operarios y técnicos & & $\mathrm{X}$ & $\mathrm{X}$ & \\
\hline Existe un sistema de señalización correcta & & $\mathrm{X}$ & $\mathrm{X}$ & \\
\hline
\end{tabular}

con las instalaciones, comprobamos la ausencia de telas mosquiteras y la carencia de limpieza y fumigación de silos en la granja de producción.

Comparando ambas granjas en cuanto a los protocolos de limpieza, desinfección y desinsectación (L+DD), observamos que la granja de producción no cuenta con registros y/o verificaciones de dichos programas, mientras que la granja de selección y multiplicación lleva un control exhaustivo de las medidas, aunque no realiza una verificación de las mismas. Respecto al control de roedores y otros animales, la granja de producción no tiene un plan documentado de desratización; durante la inspección, el veterinario comprobó la presencia de animales domésticos, si bien se mantenía un sistema de control sanitario (desparasitación y vacunación) y de acceso a las instalaciones. Por otro lado, en la granja de selección y multiplicación existe un plan documentado de desratización y no se detectó la presencia de animales domésticos. Por último, comparando los capítulos finales, observamos que los operarios de la granja de producción no llevan una vestimenta adecuada y no existe un sistema de señalización correcta de circuitos en materia de bioseguridad. Ambas granjas en este apartado carecen de un Código de Buenas Prácticas y de una formación continua de los operarios, medidas que, a nuestro juicio, deben ser incorporadas en la producción de animales de abasto.
Estudios previos muestran que la aplicación de medidas de bioseguridad aumenta considerablemente el nivel productivo de las explotaciones (Muñoz y Rouco 2006). En nuestra opinión, la verificación de las medidas de bioseguridad debe completarse con estudios que determinen la carga microbiana antes y después de la aplicación de las mismas a distintos niveles, en la propia explotación o en los animales. En este trabajo preliminar, tras la realización del cuestionario, se ha llevado a cabo un estudio microbiológico para identificar y cuantificar los microorganismos potencialmente productores de endometritis en cerdas presentes en las dos granjas seleccionadas. De los 60 hisopos uterinos analizados, en 27 (45\%, 95\% CI $[33.3 \%, 56.7 \%])$ se obtuvo crecimiento bacteriano y/o fúngico (cuadro 2). Un aspecto muy importante es que todos estos agentes identificados en cerdas sanas se han asociado a casos de endometritis clínicas, con descargas $\mathrm{y}$ problemas de infertilidad en hembras (De Winter y col 1995), por lo que podemos sugerir que todos estos microorganismos se pueden considerar potencialmente patógenos para las cerdas.

Hemos obtenido un total de 66 aislamientos (cuadro 3). Los principales géneros identificados han sido Staphylococcus spp. $(33,33 \%)$ y Aerococcus spp. $(27,27 \%)$, representados fundamentalmente por las especies Staphylococcus xylosus (16,67\%) y Aerococcus viridans (16,67\%) (cuadro 4). 
Cuadro 2. Número y frecuencia de hisopos uterinos positivos en el estudio microbiológico en ambos sistemas productivos.

Number and frequency of positive uterine swabs after microbiological study in both productive systems.

\begin{tabular}{lcccc}
\hline Explotación & $\begin{array}{c}\mathrm{N}^{\mathrm{o}} \text { hisopos } \\
\text { analizados }\end{array}$ & $\begin{array}{c}\mathrm{N}^{\mathrm{o}} \text { hisopos } \\
\text { positivos }\end{array}$ & $\begin{array}{c}\text { Frecuencia } \\
95 \% \mathrm{CI}\end{array}$ & $\mathrm{P}$ \\
\hline Producción & 20 & 13 & $\begin{array}{c}65 \%[51,3 \%, \\
78,6 \%]\end{array}$ & 0,02 \\
& & & $\begin{array}{c}35 \%[21,0 \%, \\
49,0 \%]\end{array}$ & \\
Selección & 40 & 14 & $45 \%[33,3 \%$, & \\
& & & $56,7 \%]$ & \\
TOTAL & 60 & 27 & & \\
\hline
\end{tabular}

Estos microorganismos se consideran clásicamente saprofíticos, que en animales con un buen estado sanitario no colonizarán el útero, no obstante, en condiciones adversas, pueden provocar una endometritis clínica (Rodríguez y col 1989a,b, Bara y col 1993). Además se han aislado una gran variabilidad de especies de bacterias, con una frecuencia significativamente menor, de los géneros Streptococcus $(9,09 \%)$, Enterococcus $(6,06 \%)$ y Pseudomonas $(4,55 \%)$ y hongos, entre los que cabe destacar los aislamientos de Candida, Aspergillus y Trichosporon (cuadros 3 y 4). En general, estos microorganismos, especialmente el género Streptococcus, también han sido aislados por otros autores a partir de hisopos uterinos de cerdas (Rodríguez y col 1989, y col $\left(1989^{\mathrm{b}}\right)$ obtuvieron frecuencias de aislamiento de estreptococos muy superiores $(40,6 \%)$ a las obtenidas en nuestro trabajo, posiblemente debido a que estos autores estudiaron animales con manifestaciones clínicas, siendo clásicamente aceptado que los estreptococos son los protagonistas indiscutibles en las endometritis en diferentes especies animales (Tarradas y col 1994, Luque y col 2004, Luque y col 2006). De acuerdo con Bara y col (1993), en condiciones normales estos microorganismos se pueden eliminar sin llegar a desarrollar una infección persistente que implique trastornos en la reproducción, dando lugar a cerdas totalmente sanas. Ahora bien, la localización de estos microorganismos en el endometrio puede suponer un factor de riesgo en el desarrollo de futuras endometritis. Se ha sugerido que su desarrollo depende normalmente de las medidas de higiene y manejo aplicadas en la explotación. Por lo tanto se puede establecer una relación directa entre presentación de endometritis y el nivel de bioseguridad implantado (Muñoz y Rouco 2006, Falceto y Úbeda 2008).

Cuando se comparan las dos explotaciones (cuadro 2), se observa que en la granja de producción el porcentaje de animales positivos (65\%) es el doble que en la granja de selección y multiplicación $(35 \%)(\mathrm{P}<0,05)$. No obstante, la frecuencia de aislamiento de los distintos microorganismos fue similar en ambas explotaciones, con excepción del género Enterococcus que no fue aislado en la granja de producción $(\mathrm{P}=0,012)$. Se analizó asimismo el porcentaje de infecciones mixtas (aislamiento de dos o más microorganismos en el mismo hisopo uterino), en función de la granja de origen (datos no mostrados). En nuestro estudio, en 18 de las 27 cerdas positivas $(66,67 \%)$ se aisló más de un microorganismo por muestra uterina, siendo el porcentaje de infecciones mixtas en la granja de producción significativamente superior (12/13 cerdas positivas) al obtenido en la granja de selección y multiplicación (6/14 cerdas positivas) $(\mathrm{P}=0,006)$.

Podemos concluir que la aplicación de medidas de bioseguridad que eviten la presencia de vectores y el establecimiento de estrictos protocolos de limpieza y desinfección reducen el riesgo de contaminación microbiana en el útero de cerdas tras el parto. No obstante, es necesario complementar este trabajo con estudios analíticos para identificar los factores de riesgo y protección asociados a la presencia de microorganismos potencialmente patógenos en el útero de la cerda. Estos estudios (que deben incluir un mayor número de explotaciones y muestras) permitirían calcular un modelo predictivo, para estimar la probabilidad de presentación o desaparición de este proceso en una explotación en función de las medidas de bioseguridad aplicadas.

Cuadro 3. Principales géneros bacterianos y fúngicos aislados en ambos sistemas productivos.

Main fungi and bacterial genus isolated in both productive systems.

\begin{tabular}{|c|c|c|c|c|c|}
\hline \multirow{2}{*}{ Género } & \multirow{2}{*}{$\begin{array}{c}\text { Granja Producción } \\
\text { Número }(\%)\end{array}$} & \multirow{2}{*}{$\frac{\text { Granja Selección }}{\text { Número }(\%)}$} & \multicolumn{3}{|c|}{ TOTAL } \\
\hline & & & $P$ & Número (\%) & $95 \% \mathrm{CI}$ \\
\hline Staphylococcus spp & $15(34,88)$ & $7(30,46)$ & 0,78 & $22(33,33)$ & {$[21,9 ; 44,7]$} \\
\hline Aerococcus spp & $14(32,56)$ & $4(17,39)$ & 0,25 & $18(27,27)$ & {$[16,5 ; 38,0]$} \\
\hline Streptococcus spp & $2(4,65)$ & $4(17,39)$ & 0,17 & $6(9,09)$ & {$[2,16 ; 16,0]$} \\
\hline Enterococcus spp & - & $4(17,39)$ & 0,012 & $4(6,06)$ & {$[0,30 ; 11,8]$} \\
\hline Pseudomonas spp & $3(6,98)$ & - & 0,54 & $3(4,55)$ & {$[0,00 ; 9,57]$} \\
\hline Otros & $9(20,93)$ & $4(17,39)$ & 1 & $13(19,70)$ & {$[10,1 ; 29,3]$} \\
\hline TOTAL & 43 & 23 & & 66 & \\
\hline
\end{tabular}


Cuadro 4. Microorganismos aislados a partir de los hisopos uterinos analizados en ambos sistemas productivos. Microorganisms isolated from uterine swabs analyzed from both production systems.

\begin{tabular}{lccc}
\hline Especie identificada & $\begin{array}{c}\text { Granja Producción } \\
\text { Número }(\%)\end{array}$ & $\begin{array}{c}\text { Granja Selección } \\
\text { Número }(\%)\end{array}$ & $\begin{array}{c}\text { Total Granjas } \\
\text { Número }(\%)\end{array}$ \\
\hline Aerococcus viridans & $10(23,26)$ & $1(4,35)$ & $11(16,67)$ \\
Aerococcus urinae & $4(9,30)$ & - & $4(6,06)$ \\
Aerococcus spp & - & $3(13,04)$ & $3(4,55)$ \\
Staphylococcus xylosus & $11(25,60)$ & - & $11(16,67)$ \\
Staphylococcus aureus & $2(4,66)$ & $1(4,35)$ & $3(4,55)$ \\
Staphylococcus chromogenes & - & $3(13,04)$ & $3(4,55)$ \\
Staphylococcus auricularis & - & $1(4,35)$ & $1(1,52)$ \\
Staphylococcus hominis & $1(2,32)$ & - & $1(1,52)$ \\
Staphylococcus hyicus & - & $1(4,35)$ & $1(1,52)$ \\
Staphylococcus lentus & $1(2,32)$ & - & $1(1,52)$ \\
Staphylococcus sciuri & - & $1(4,35)$ & $1(1,52)$ \\
Streptococcus group L & - & $3(13,04)$ & $3(4,55)$ \\
S. dysgalactiae ssp equisimilis & - & $1(4,35)$ & $1(1,52)$ \\
Streptococcus porcinus & $1(2,32)$ & - & $1(1,52)$ \\
Streptococcus suis & $1(2,32)$ & - & $1(1,52)$ \\
Enterococcus faecalis & - & $3(13,04)$ & $3(4,55)$ \\
Enterococcus spp & - & $1(4,35)$ & $1(1,52)$ \\
Pseudomonas fluorescens & $3(6,98)$ & - & $3(4,55)$ \\
Bordetella spp & $3(6,98)$ & - & $3(4,55)$ \\
Candida spp & $1(2,32)$ & $2(8,70)$ & $3(4,55)$ \\
Corynebacterium group F-1 & $2(4,66)$ & - & $2(3,03)$ \\
Escherichia coli & - & $2(8,70)$ & $2(3,03)$ \\
Aspergillus fumigatus & $1(2,32)$ & - & $1(1,52)$ \\
Ewingella americana & $1(2,32)$ & - & $1(1,52)$ \\
Trichosporon asahii & $1(2,32)$ & & $1(1,52)$ \\
Total & $43(100)$ & & $66(100)$ \\
\hline & & $230)$ &
\end{tabular}

\section{RESUMEN}

Se entiende por bioseguridad todas las medidas aplicadas que tienen como objetivo minimizar los riesgos sanitarios en una explotación ganadera, e incluye medidas relacionadas con las instalaciones y el manejo. La eficacia de estas medidas debe reflejarse en una disminución de la carga microbiana en las distintas fases productivas. En este trabajo se pretende evaluar si el conjunto de medidas aplicadas influye en la contaminación microbiana uterina tras el parto de cerdas sanas. Para ello se estudiaron dos granjas porcinas, una granja de producción y otra de selección y multiplicación, donde se completó un cuestionario de medidas de bioseguridad y realizó un estudio microbiológico de hisopos uterinos de cerdas postparto. Se han analizado un total de 60 animales, de los que 27 (45\%) han resultado positivos, encontrando diferencias significativas entre la granja de producción y la de selección y multiplicación $(\mathrm{OR}=3,44$, IC 95\%, 1,135-11,047). La frecuencia de colonización fue del $65 \%$ CI $[51,3 \%, 78,6 \%]$ y $35 \%$ CI $[21 \%, 49 \%]$ en la granja de producción y en la granja de selección, respectivamente $(\mathrm{P}=0,02)$. Se han obtenido 66 aislamientos, representados fundamentalmente por Staphylococcus spp $(33,33 \%)$ y Aerococcus spp $(27,27 \%)$, aunque se han aislado también otras especies incluidas en los géneros Streptococcus $(9,09 \%)$, Enterococcus $(6,06 \%)$ y Pseudomonas $(4,55 \%)$, así como diferentes especies de hongos. La frecuencia de aislamiento de los diferentes microorganismos fue similar en ambas granjas, a excepción del género Enterococcus, que no fue aislado en la granja de producción $(\mathrm{P}=0,01)$. El cuestionario de bioseguridad mostró algunas diferencias en las medidas aplicadas en la granja de selección y multiplicación sobre la de producción, lo cual unido a la mayor colonización uterina observada en esta última nos lleva a plantear una hipótesis preliminar sobre los posibles factores de riesgo asociados a este proceso, destacando la ausencia de medidas que eviten la presencia de vectores y el establecimiento de estrictos protocolos de limpieza y desinfección.

\section{AGRADECIMIENTOS}

Se agradece la participación de D. Francisco Colino Rivas, Director de Producción y Sanidad de la Empresa de Selección Genética Topigs Ibérica, tanto en la recolección y envío de muestras así como en completar la encuesta de bioseguridad, necesarios para la realización de este trabajo. 


\section{REFERENCIAS}

Bara MR, MR McGowan, D O’Boyle, RDA Cameron. 1993. A study of the microbial flora of the anterior vagina of normal sows during different stages of the reproductive cycle. Aust Vet J 70, 256-259.

Boletín Oficial del Estado. 2000. Real Decreto 324/2000 del 8-03-2000 por el que se establecen normas básicas de ordenación de las explotaciones porcinas. Boletín Oficial del Estado 58, 9505-9512.

De Winter PJ, M Verdonsk, A de Kruif, L Devriese, F Haesebrouck. 1995. Bacterial endometritis and vaginal discharge in the sow: prevalence of different bacterial species and experimental reproduction of the syndrome. Anim Reprod Sci 37, 325-335.

Falceto MV, JL Úbeda. 2008. Origen y control del Síndrome de la Descarga Vulvar en la cerda. Av Tecnol Porc 7-8, 4-24.

Fthenakis GC. 1994. Prevalence and aetiology of subclinical mastitis in ewes of Southern Greece. Small Ruminant Res 13, 293-300.

Luque I, R Cobo, A Maldonado, RJ Astorga, A Carbonero, I GarcíaBocanegra, C Tarradas. 2004. Endometritis Equina I: Aislamiento e Identificación de especies del género Streptococcus. Equinus 4, 39-54.
Luque I, JF Fernádez-Garayzabal, V Blume, A Maldonado, R Astorga, C Tarradas. 2006. Molecular typing and anti-microbial susceptibility of clinical isolates of Streptococcus suis ssp. zooepidemicus from Equine Bacterial Endometritis. J Vet Med B 53, 451-454.

Muñoz A, A Rouco. 2006. Análisis financiero de la inversión en bioseguridad. Porci 96, 45-54

Quiles A. 2008. Bioseguridad en las explotaciones porcinas. Ediporc $114,20-34$

Quiles A, ML Hevia. 2006. Limpieza y desinfección: tecnología todo dentro/todo fuera. Producción Animal 6, 4-27.

Rodríguez A, MV Latre, L Tarrafeta, JA Bascuas, P Gaspar. 1989ª Aislamiento de microorganismos bacterianos aerobios del aparato reproductor de cerdas (ovario, oviductos y cuernos uterinos) afectadas de procesos patológicos. Anaporc 76, 57-62.

Rodríguez A, L Tarrafeta, JA Bascuas, J Ducha, J González. 1989. Microorganismos bacterianos aerobios del flujo vaginal de cerdas. Anaporc 76, 75-78.

Tarradas MC, A Arenas, A Maldonado, A Perea. 1994. Infecciones por Streptococcus suis en ganado porcino. Anaporc 131, 4-29 